\title{
An Ordinance-Tweet Mining App to Disseminate Urban Policy Knowledge for Smart Governance
}

\author{
Christina Varghese ${ }^{1}$, Aparna S. Varde ${ }^{1,3(\bowtie)}$, and $\mathrm{Xu} \mathrm{Du}^{2,3}$ \\ 1 Department of Computer Science, \\ Montclair State University, Montclair, NJ, USA \\ \{varghesecl, vardea\}@montclair.edu \\ 2 Department of Earth and Environmental Science, \\ Montclair State University, Montclair, NJ, USA \\ dux3@montclair.edu \\ 3 Environmental Science and Management PhD Program, \\ Montclair State University, Montclair, NJ, USA
}

\begin{abstract}
This paper focuses on how populations by the use of technology, more specifically an app, can comprehend the enactment of ordinances (local laws) in an urban area along with their public reactions expressed as tweets. Furthermore, they can understand how well the area is developing and enhancing as a Smart City. The main goal of this research is to develop an Ordinance-Tweet Mining App that disseminates the results of analyzing ordinances and tweets about them, especially related to Smart City Characteristics such as Smart Environment, Smart Mobility etc. This app would be beneficial to various users such as environmental scientists, policy makers, city committees as well as the common public in becoming more aware of legislative bodies, and possibly contributing in different aspects to make the urban area improve as a Smart City. This work fits the realm of Smart Governance due to transparency via public involvement.
\end{abstract}

Keywords: App development - Human Computer Interaction (HCI) -

Legislative information $\cdot$ Opinion mining $\cdot$ Twitter data $\cdot$ Smart cities $\cdot$ Urban policy

\section{Introduction}

The paradigm of Smart Governance leverages transparency through public inclusion. This is gaining impetus with Smart Cities [1,2]. It is thus important to disseminate knowledge on urban policy to the public. Ordinances (local laws) are often publicly available, e.g. [3], yet many residents view them as impervious due to complex legalese. Thus, ordinances need mining to discover knowledge that a wide spectrum of users can comprehend. A related aspect is public opinion on social media. It is important to gauge public reactions on issues related to ordinances by opinion mining. The results of mining ordinances and their public reactions need dissemination to be easily accessible and understandable. This is the focus of our overall research $[4,5]$. 
We address ordinances from publicly available sites, and reactions to them expressed on Twitter. We conduct mining on these to discover knowledge on how well the ordinances enhance the given area into a Smart City, and to what extent the public is satisfied with the ordinances.

We conduct such ordinance and tweet mining, guided by commonsense knowledge (CSK) [6] to capture subtle human reasoning. We use CSK sources, along with the well-known word2vec [7] for ordinance-tweet mapping, and with sentiment polarity classification for mining. Based on this, we develop an Ordinance-Tweet Mining App, to disseminate the analysis. In this paper, we describe the app development using principles from HCI (Human Computer Interaction), e.g. [8, 9]. We explain the mining of ordinances and tweets leading to useful legislative information disseminated by the app. This app provides QA (Question Answering) on interesting issues in urban policy. It is user-friendly, including interactive graphs and FAQs (Frequently Asked Questions) that facilitate comprehension by the public and experts. Targeted users, including the common public and domain experts in environmental management evaluate this app. In this paper, we focus on disseminating the results of mining NYC ordinances available on its public legislative council website [3]. We collect tweets from NYC through location-based data available on Twitter. We consider NYC since it is the financial capital of the USA, one of the major metropolitan cities in the world and a leading Smart City (see Fig. 1) [3, 10]. As per world rankings, NYC is among the top 25 smart cities worldwide [11]. This is a good achievement. Yet there is scope for enhancement. We address this in the mining of ordinances and tweets, and in the corresponding app.

We present the development and experimentation of our Ordinance-Tweet Mining App herewith. To the best of our knowledge, ours is the first app disseminating the outcomes of ordinance-tweet mining, leveraging HCI. This app contributes to Smart Governance by making information on urban policy ubiquitous and comprehensible.

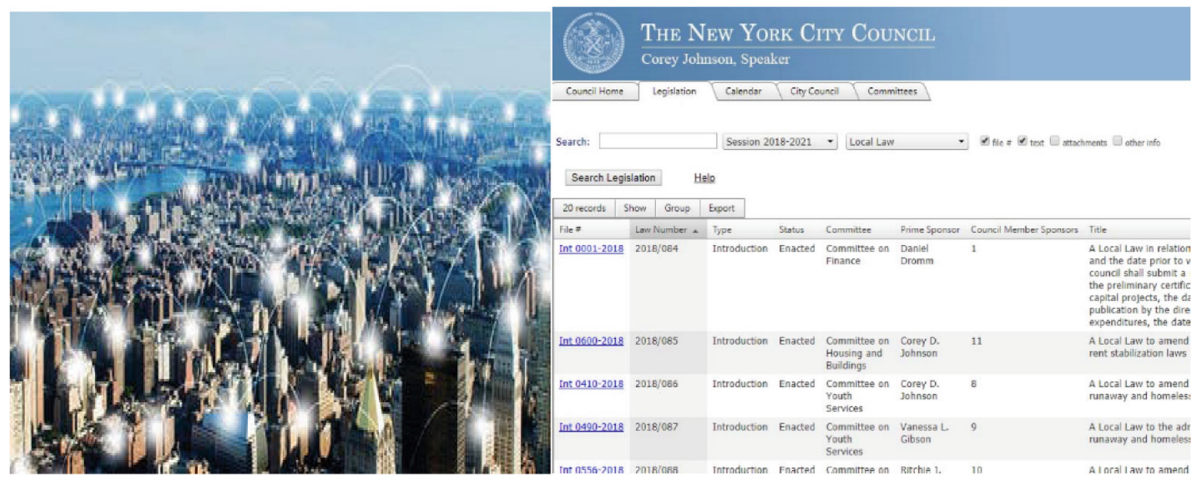

Fig. 1. NYC (New York City) as a prominent smart city [left] and NYC council website [right] 


\section{Overview of Ordinance and Tweet Mining}

In our earlier works, we propose methods for mining of ordinances from websites [4, 12] and furthermore, the mining of public opinions about them expressed on Twitter [5, $13,14]$. We use these methods for ordinance-tweet mining within NYC and conduct evaluation as included in our papers [4, 5, 12-14]. As stated in [5, pp. 1721-1722], "an important focus in our work is to determine to what extent such ordinances contribute to establishing the relevant urban region as a Smart City. Hence, we categorize ordinances based on their pertinent Smart City Characteristics (SCCs). We aim to connect ordinances to relevant tweets by drawing on their semantic relatedness. This is nontrivial, as ordinances and tweets both involve highly intricate and rather heterogeneous natural language, so simple keyword matching does not suffice. We propose a two-step approach for mapping that exploits the transitive nature of the connection between ordinances and tweets considering their relationship with SCCs. Specifically, the transitive property we invoke is that: if the ordinance relates to a given SCC and any tweet relates to the same SCC, then the ordinance bears a connection to the tweet. This approach is proposed because classical sources of SCC data e.g., [1,2] are finite and are restricted to a limited set of identifying features that can be relied upon for mapping. Thus, this transitive approach is more feasible than attempting to directly relate a seemingly infinite number of tweets to ordinances from various websites. As a first step, we discover connections between SCCs and ordinances using classical SCC sources [2] guided by commonsense knowledge (CSK) from web-based repositories $[15,16]$. In the second step, we consider the mapping of tweets to SCCs, again drawing on such CSK. This approach then enables us to directly relate ordinances and the tweets to the pertinent aspects of Smart Cities and also sets the stage for sentiment polarity classification" [5, pp. 1721-1722]. Based on this, our mapping algorithm is Algorithm 1, as presented herewith [5].

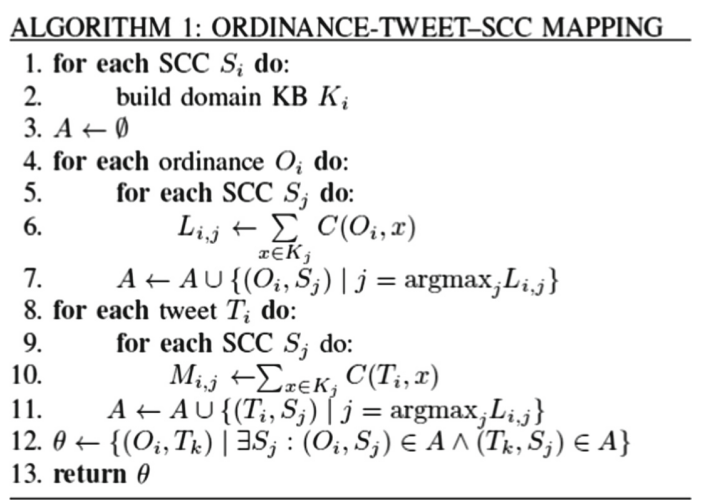

(Source: [5])

As stated in [13, pp. 841-842], "we conduct sentiment analysis to discover knowledge specifically with respect to opinion mining of tweets on ordinances. This is conducted after the mapping of ordinances to tweets. The primary database used for 
Sentiment Analysis in this work is SentiWordNet [17]. This is an enhanced version of the CSK source WordNet [15]. It groups words into synonym sets (synsets) annotated by how positive the terms are. Accordingly, words are classified as positive, negative or neutral based on polarity of terms. In SentiWordNet, different meanings exhibited by the same word can have different sentiment scores. For example, the word estimable when relating to computation has a neutral score of 0.0 , while the same word in the sense of deserving respect is assigned a positive score of 0.75 . The process we deploy for sentiment analysis of tweets constitutes a semi-supervised learning method using SentiWordNet. Through this, subtle human judgment through commonsense in understanding emotions is embodied in the mining processes with specific reference to context" [13, pp. 841-842]. Accordingly, our algorithm for polarity classification of tweets is Algorithm 2 as presented next [13].

\section{ALGORITHM 2: TWEET POLARITY CLASSIFICATION}

1. for each tweet $t_{i}$ do:

2. if not $\left(t_{1}\right.$ relevant according to $\left.\mathrm{SCC} \mathrm{KB}\right)$ :

$3 . \quad$ continue (with next tweet)

4. $\quad$ map $t_{i}$ to ordinances using Algorithm 1

5. $\quad W_{i} \leftarrow$ set of words in $t_{i}$

6. $\quad$ for each $w \in W_{i}$ do:

7. $\quad s_{w} \leftarrow$ polarity score of $w$ in SentiWordNet

8. $\quad s_{i} \leftarrow \sum_{w \in W_{i}} s_{w}$

9. return final polarity scores $s_{i}$ for relevant $t_{i}$

(Source: [13])

As further stated in [13, pp. 841-842], "based on this algorithm, we classify thousands of tweets that we obtain from Twitter. Note that the selection of relevant tweets and also the mapping of tweets to their respective ordinances is guided by CSK. We construct SCC-based Domain KBs (Domain-Specific Knowledge Bases) [5, 13, 14] derived from WebChild [16] and WordNet [15], to filter out unwanted tweets as a first step, followed by the mapping of tweets to relevant ordinances using SCCs as a next step" [13, pp. 841-842]. We start mapping groups of ordinances to tweets [5, 13], and then connect them with each other at finer levels of granularity [14] by mapping individual ordinances to tweets. This is via ordinance KBs in addition to SCC KBs, incorporating pragmatics and semantics through CSK and domain knowledge respectively [14]. The mining in our work is on ordinances and tweets from NYC, using a public council site [3] and Twitter location-based data. The results of the SCCbased ordinance analysis and tweet polarity classification comprise significant inputs for building the Ordinance-Tweet Mining App. We now describe its design process.

\section{Approach for App Design}

With the advancement in technology over the years, we are now benefiting from the "The Digital Age" which gave rise to the Internet and various mobile devices. This has digitized many institutions over the past few decades with governments processing applications via e-government websites that help people process their applications 
faster and avoid long wait periods. Retail stores today have e-commerce websites that help them reach a global audience. This progress has evolved the manner in which we do business on a higher scale. New technologies emerging in AI will help improve urban policies significantly [18], especially with respect to outreach initiatives. Users today often wish to have ubiquitous access to information. This leads to the development of apps. Accordingly, in the realm of e-government, an app for disseminating the results of ordinance-tweet mining is useful.

Based on this background and the overview of our ordinance and tweet mining research, we explain our proposed approach to design an Ordinance-Tweet Mining App. We comprehensively use principles from Human Computer Interaction (HCI) [8, $9,19,20]$ to create a user experience that would be all encompassing for various users. An important concept in HCI is Fitt's Law [9, pp. 518-519], i.e., " $T=k \log _{2}$ $(D / S+1.0)$ where $T=$ time to move the pointer to a target, $D=$ distance between the pointer and the target, $S=$ size of the target, $k$ is a constant of approximately $200 \mathrm{~ms} /$ bit". We incorporate Fitt's Law in our app design. Thus, we design items in the app such that they are big enough to enable users to spot them fast, especially as navigation proceeds further from the opening screen. Yet, these items are small enough to fit on the required screens, and hence users do not need to spend much time while searching.

For the layout of the app, we use another HCI concept, i.e. "mockup designs". Mockups are multiple designs created by interviews with "stakeholders" that give an overview of the blueprint of the app [8]. Stakeholders in HCI terminology are various influential groups such as domain experts who have a thorough knowledge of the field; students/working professionals involved in providing data; and end-users ranging from novice to expert, and casual to frequent [8]. Among the mockups, we select the best designs for the app layout and screens. Other HCI aspects we incorporate are the simplicity and efficiency of the interface [20]. These include navigation, the time spent to find an answer to a question asked by the user, and the analytical graphs displayed. Considering HCI, some principles used in the app adhere to the guidelines of Google's material design [19] to ensure that the app is up to date with the current and latest software, and to warrant that the app runs smoothly without errors or bugs. Another HCI aspect is a "metaphor" [9]. The term metaphor refers to conceptualization of actions, typically for the interface. For example, a shopping cart is an interface metaphor used for checkout in online shopping. We incorporate metaphors in our app for various purposes, e.g. to depict different ordinance departments and smart city characteristics.

Good design of this app entails navigating pages without spending too much time on locating the options that the users need [20]. Based on HCI principles, Fig. 2 illustrates the navigation of the app in an efficient manner to access the given information. For details, please see [21]. This navigation keeps the users engaged and alert. Another important factor is the specific usage to assess the emotions of users while navigating the app and seeing the results. In order to incorporate this, we make sure that the intermediate pages and end-results focus on inclusion and interactive design elements [19]. 


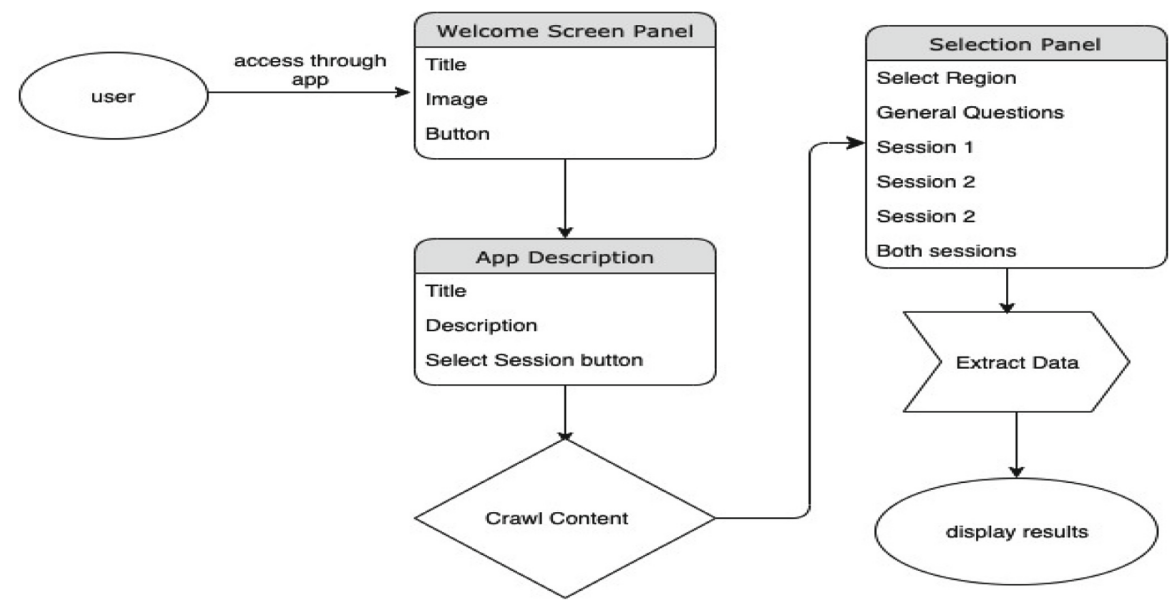

Fig. 2. App navigation flowchart

\section{Implementation of the App}

For the implementation of the Ordinance-Tweet Mining App, we use Android Studio $[22,23]$ as the Integrated Development Environment (IDE). Android Studio provides useful features that make the actual programming of app very convenient and efficient. The IDE facilitates development and use for anyone ranging from a beginner to a fullfledged software engineer [24]. Some features of Android Studio are:

- Code Completion

- Creation of Templates

- Instant App Run

- Fast Emulator

- Smart Code Editor

- Kotlin Programming Language Support

Based on these features, we implement the Ordinance-Tweet Mining App deploying Android Studio. Figure 3 illustrates the implementation process using UML for Android Development [23] with a self-explanatory diagram. For a more detailed explanation, please see [21]. Given this implementation, Fig. 4 shows a snapshot of the app layout. The left screen in the figure serves as a landing and welcome page with a call to action button labeled "Get Started". This lets the users know that they can access the app and navigate to the desired location. The center screen gives a brief description of the app. Once the users are ready to select an option, they can click on "Select Ordinance Session". The right screen serves as an action sheet. Action sheets are helpful whenever there are multiple actions [20]. They work well on this screen, since it shows the different options for the region and the sessions that the users can select. 


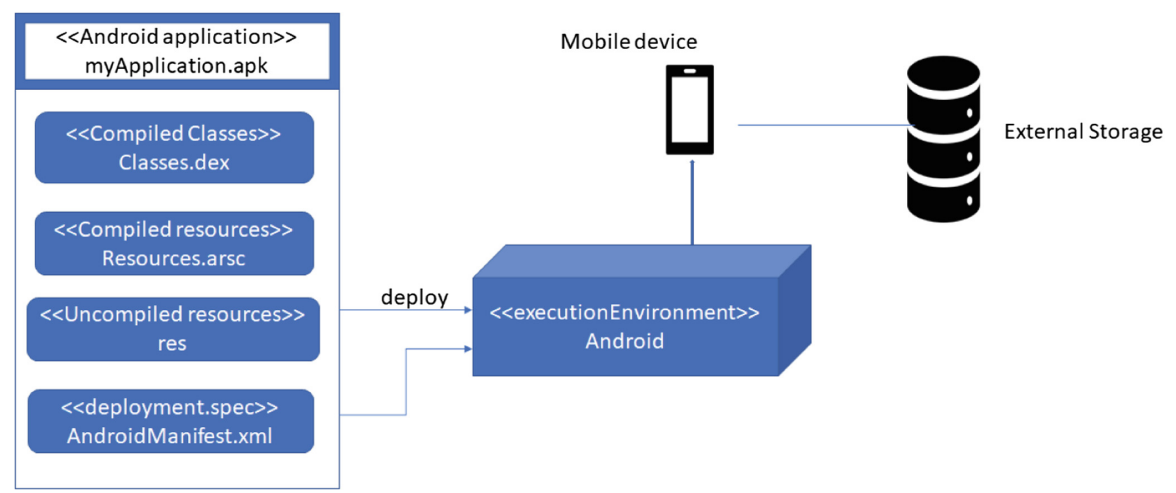

Fig. 3. Implementation process of the app using the Android platform

Figure 5 depicts the screens with FAQs and corresponding results of ordinance mining for the NYC ordinance passing sessions considered [3], i.e. Session 1 (20062009) and Session 2 (2010-2103). The leftmost screen includes general questions that users may have for example, "What is a smart city?" "What specific characteristics does a smart city have?" and so on. The other screens depict the outputs from the mining of the specific sessions and the combined results from mining both the sessions.

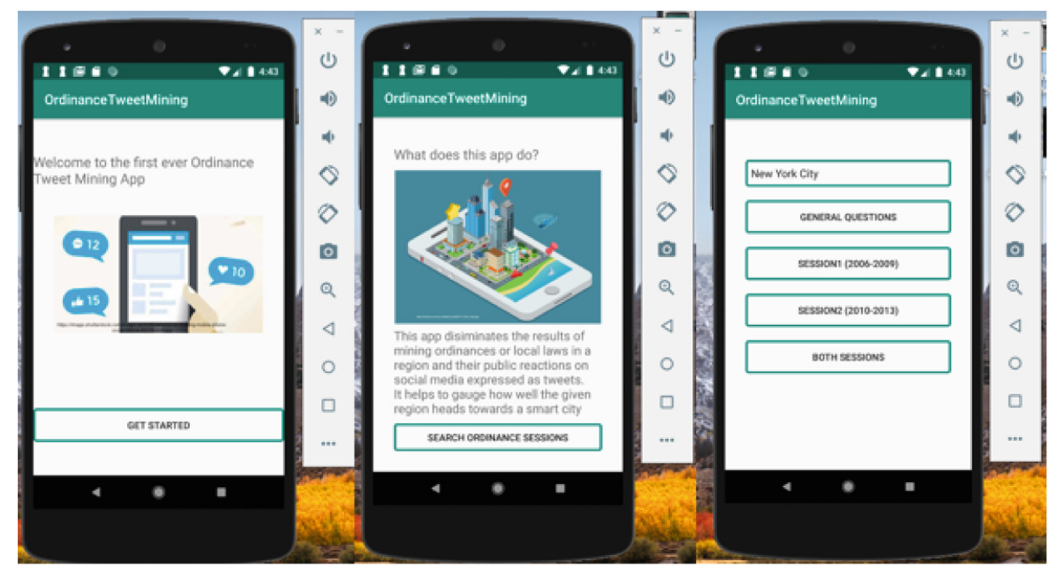

Fig. 4. Layout screens of the Ordinance-Tweet Mining App 


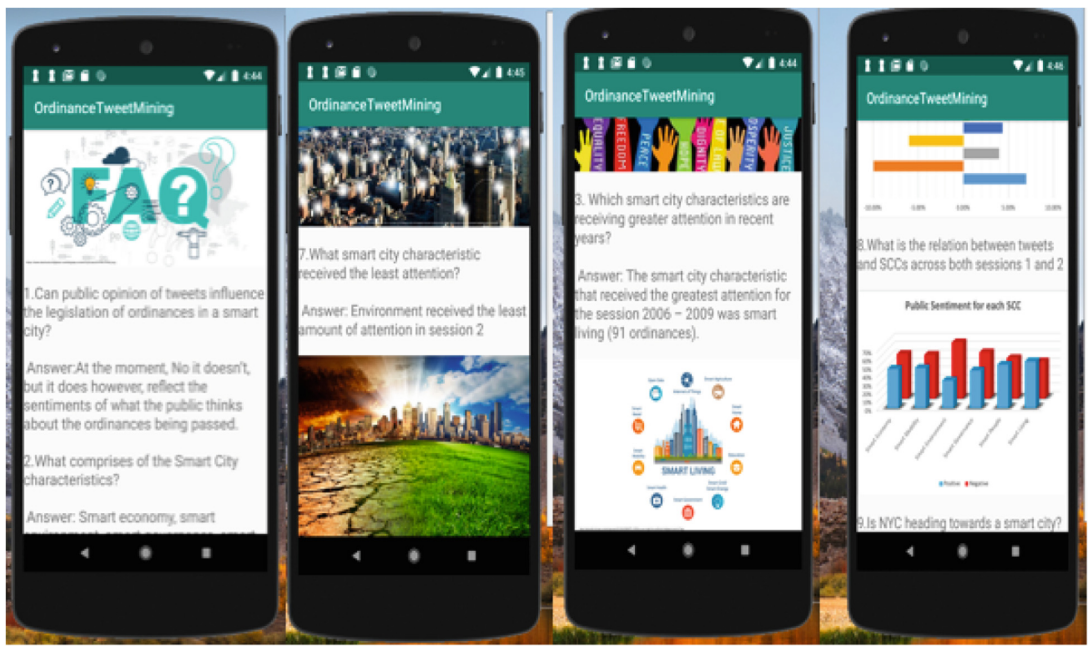

Fig. 5. FAQs for various selection categories in the app

\section{Experiments and Discussion}

In order to evaluate the app, we conduct user surveys [21]. We create survey questions using a Likert Scale format [9]. We summarize the results in Figs. 6, 7 and 8. These encompass the feedback of 34 participants with an assortment of computer and environmental scientists, students, lawyers, policymakers and researchers. The main questions are as follows with responses on a scale of 1-5 [1: Strongly Agree... 5: Strongly Disagree]

- Q1: Do you find this app, quick and easy to use?

- Q2: Does this work increase public awareness of urban policy?

- Q3: Do you feel NYC is getting better as a smart city?

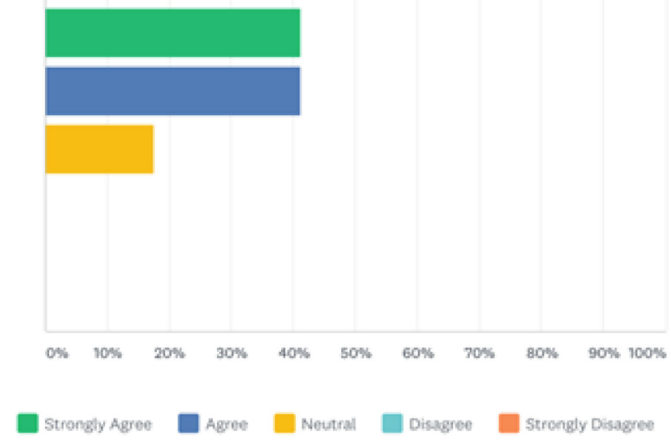

Fig. 6. Responses to Q1: "Do you find the app quick and easy to use?" 


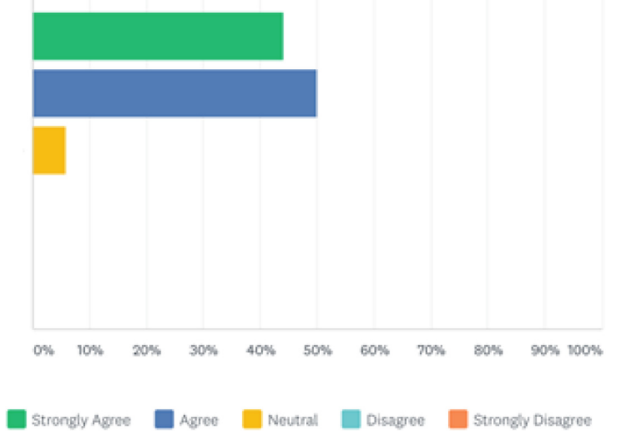

Fig. 7. Responses to Q2: "Does this work increase public awareness of urban policy?"

As seen in these figures, the user survey results indicate favorable responses towards this app. After collecting the data from the surveys, it is evident that the app is feasible for use and makes users more aware of urban policy. Many users feel that NYC is getting better as a smart city while some are neutral. In addition to the Likert scale evaluation, some comments included by the users in the survey are as follows.

- "This can be useful in courses on urban policy"

- "This app looks great - simple and informative is what New Yorkers need. I would have liked to have access to links to the researcher's published work, if available or maybe lists of smart city ordinances as a resource."

- "Very good usage of graphics, makes data easier to understand"

- "Great work you are doing. Well-done"

- "I am interested in understanding the technology behind this product"

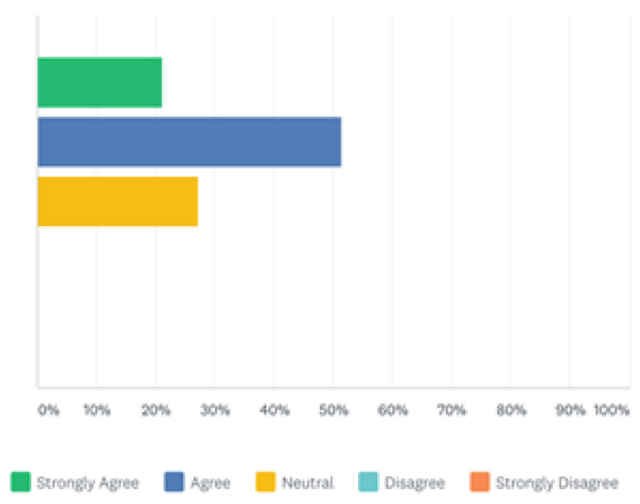

Fig. 8. Responses to Q3: "Do you feel NYC is getting better as a smart city?"

Based on these survey results and the general feedback received from the users we can infer that the work on extracting ordinance data and conducting sentiment analysis 
through mining of tweets have proven useful by reaching a larger audience and by disseminating information that may not have been readily available to the public earlier. Please note that our research on ordinance-tweet mining as well as the development of this app both constitute pioneering work in the area, to the best of our knowledge. Hence, we do not conduct comparative studies for the mining or the app development.

All this work is in line with the recent concept of greater awareness and more transparency in governance. Some local celebrities have played their part by influencing laws that improve society. Tim Tebow, a quarterback for NY Jets inspired South Carolina legislators to pass the Equal Access to Interscholastic Activities act in May 2012. Also known as the "Tim Tebow Law" [25], this allows homeschooled children to participate in public school extracurricular activities. Candy Lightner founded the "Mothers against Drunk Driving (MADD)" organization to ensure sterner laws against drunk driving [26]. Due to the media attention this organization received with Candy's story, it raised more awareness of driving responsibly. Likewise, our Ordinance-Tweet Mining App, the first of its kind, is likely to play an important role in making the public more aware of legislative policies and take action needed for enhancement. This concept fits the realm of Smart Governance [1, 2], a characteristic of Smart Cities that leverages greater transparency in governing processes via more public involvement.

\section{Related Work}

Social media users generate massive amounts of information in their daily life. Scientists consider that as valuable data for various studies on urban policy, traffic, energy conservation, climate change, disaster management etc. Social media text mining is therefore a powerful tool to extract useful knowledge.

$\mathrm{Gu}$ et al. [27] propose a method to use tweets for gathering road incident information. They build an API (Application Programming Interface) to compare incidents recorded on social media and in traditional databases. This not only validates existing incident information but also finds new incidents, thus supplementing the data in databases. Gandhe et al. [28] conduct sentiment analysis of data from Twitter on political scenarios, urban events etc. As stated in [28, p. 57], the "proposed approach entails a hybrid learning method for classification of tweets based on a Bayesian probabilistic method for sentence level models given partially labeled training data". The advantage is that the approach is semi-supervised, and works even with partly labeled data. Nuortimo [29] studies social media data from multiple platforms to understand public reactions for a system called Case Carbon Capture and Storage, to control carbon dioxide emissions. Results show that the overall reactions are positive. This study indicates that social media mining could be a great tool to measure public awareness and acceptance for topics related to energy and climate. Huang et al. [30] assess disaster analysis of historical and future events. They gather social media, remote sensing and Wikipedia data, performing spatial analysis and social media mining. Their results show that social media mining enhances disaster analysis and provides real-time tracking. 
All these works demonstrate the potential of social media mining. Accordingly, if an app disseminates knowledge from such mining for public outreach, it would have broader impacts on sustainability and Smart Cities [1, 2, 11], especially with reference to Smart Governance [2]. It would foster building other related apps. The sharing of data via such apps would benefit pertinent research. Various useful mobile apps for Androids exist in the literature, as described in recent work [31]. Our design and development of the Ordinance-Tweet Mining App contributes to this overall realm.

Various aspects of AI can make an impact on Smart Cities as surveyed in the literature [2, 6, 18, 32-34]. AI can help record car activity, foot traffic, types of shoppers that go to different retailers, their preferences, availability of parking spots etc. These are minor details yet they make big impacts to create efficient solutions [18]. AI can contribute to autonomous and semi-autonomous driving through incorporation of CSK-based techniques for enhanced decision-making [6, 32]. AI can play a role in augmenting object detection for Smart Mobility in Smart Cities with neural models, deep learning, CSK and adversarial datasets [6, 33, 35]. Another major aspect is AI in lighting. NYC has bright lights, which imply significant energy consumption. Thus, if lamppost design occurs with sensors, these can adjust their brightness depending on the amount of traffic within the area. In some cities such as Amsterdam, canal lights dim and brighten based on pedestrian usage [2]. Likewise, AI can contribute to several aspects of Smart Cities [34]. Our work in this paper is a step in this direction, using HCI-based app design and disseminating the results of mining ordinances along with their public reactions. Hence, this paper makes an impact on Smart Governance in Smart Cities.

\section{Conclusions}

This paper addresses the development of an Ordinance-Tweet Mining App that disseminates knowledge discovered by mining ordinances in a given region and tweets about them, especially relevant to Smart Cities. Through this app, users from various backgrounds can obtain quick and easy access to legislative information. Via the app the public can make better decisions and contributions, e.g. by understanding policies and public reactions, they can participate city council committees, or support their region through financial means and community outreach. In addition, this app can provide decision support to lawmakers by providing ubiquitous information, and can enhance the scope of study for researchers through future issues emerging from the work here. To the best of our knowledge, ours is the first ever Ordinance-Tweet Mining App. While this app focuses on NYC in particular, it can foster the development of similar or related apps for other cities, by reuse of the approaches and data with modification.

Future work includes embedding intricate NLP (Natural Language Processing) along with semantics and pragmatics in order to facilitate direct QA (Question Answering) beyond static FAQs (Frequently Asked Questions) and keywords. This would encompass advances in the field of CSK (Commonsense Knowledge) to fathom the QA text and give enhanced responses in the app based on the mining results. This 
constitutes part of our ongoing research. In general, our work in this paper makes a broader impact on Smart Governance.

\section{References}

1. IEEE Smart Cities: What Makes a City Smart (2020). https://smartcities.iee.org/

2. TU Wien: European Smart Cities, Technical Report, Vienna University of Technology, Austria, August 2015

3. The New York City Council: Legislative Research Center (2018). http://legistar.council.nyc. gov/

4. Du, X., Liporace, D., Varde, A.: Urban legislation assessment by data analytics with smart city characteristics. In: IEEE UEMCON, pp. 20-25, October 2017

5. Puri, M., Du, X., Varde, A., de Melo, G.: Mapping ordinances and tweets using smart city characteristics to aid opinion mining. In: WWW Companion, pp. 1721-1728, April 2018

6. Tandon, N., Varde, A., de Melo, G.: Commonsense knowledge in machine intelligence. ACM SIGMOD Rec. 46(4), 49-52 (2017)

7. Mikolov, T., Sutskever, I., Chen, K., Corrado, G., Dean, J.: Distributed representations of words and phrases and their compositionality. In: NIPS, pp. 3111-3119, December 2013

8. Harper, R., Rodden, T., Rogers, Y., Sellen, A.: Being Human: Human-Computer Interaction in the Year 2020. Microsoft Research (2008)

9. Rogers, Y., Sharp, H., Preece, J.: Interaction Design: Beyond Human-Computer Interaction, 4th edn. Wiley, Hoboken (2015). ISBN 978-1-119-02075-2

10. NYC Smart City: Eventa. https://www.eventa.us/events/new-york-ny/nyc-smart-city-trek

11. The EasyPark Group: 2017 Smart Cities Index (2017). https://easyparkgroup.com/smartcities-index

12. Du, X., Varde, A., Taylor, R.: Mining ordinance data from the web for smart city development. In: DMIN, pp. 84-90. CRSEA Press, July 2017. ISBN 1-60132-453-7

13. Puri, M., Varde, A., Du, X., de Melo, G.: Smart governance through opinion mining of public reactions on ordinances. In: IEEE ICTAI, pp. 838-845, November 2018

14. Puri, M., Varde, A., Dong, B.: Pragmatics and semantics to connect specific local laws with public reactions. In: IEEE Big Data, pp. 5433-5435, December 2019

15. Miller, G.: WordNet: a lexical database for English. Commun. ACM 38(1), 39-41 (1995)

16. Tandon, N., de Melo, G., Suchanek, F., Weikum, G.: WebChild: harvesting and organizing commonsense knowledge from the web. In: ACM WSDM, pp. 523-532, February 2014

17. Baccianella, S., Esuli, A., Sebastiani, F.: SentiWordNet 3.0: an enhanced lexical resource for sentiment analysis and opinion mining. In: LREC, May 2010

18. Dirican, C.: The impacts of robotics and artificial intelligence on business and economics. Soc. Behav. Sci. 195, 564-573 (2015)

19. Soni, S.: Google Material Design's Impact on Mobile App Design, February 2019. https:// appinventiv.com/blog/mobile-app-designers-guide-on-material-design/

20. So, Y.: Designing for Mobile Apps: Overall Principles, Common Patterns, and Interface Guidelines, May 2012. https://medium.com/blueprint-by-intuit/native-mobile-app-designoverall-principles-and-common-patterns-26edee8ced10

21. Varghese, C., Varde, A.: Disseminating results of mining ordinances and their tweets by Android app development. Technical report, Montclair State University, December 2019

22. Android Developers: The Android Studio. https://developer.android.com/studio

23. Android Application: UML Deployment. https://www.uml-diagrams.org/android-applicati on-uml-deployment-diagram-example.html 
24. ADMEC Institute: Top 10 Features of Android, July 2018. https://www.admecindia.co.in/ blog/top-10-features-android-studio-developers-not-miss

25. The Week: 7 Celebrities Who Inspired New Laws. Business Insider, September 2012. https://www.businessinsider.com/7-laws-named-for-celebrities-2012-9\#tim-tebow-2

26. O'Connell, C.: 15 Ordinary People Who Changed History. Reader's Digest: https://www.rd. com/true-stories/inspiring/inspiring-stories-9-ordinary-people-who-changed-history/

27. Gu, Y., Qian, Z., Chen, F.: From Twitter to detector: real-time traffic incident detection using social media data. Transp. Res. Part C: Emerg. Technol. 67, 321-342 (2016)

28. Gandhe, K., Varde, A., Du, X.: Sentiment analysis of Twitter data with hybrid learning for recommender applications. In: IEEE UEMCON, pp. 57-63, October 2018

29. Nuortimo, K.: Measuring public acceptance with opinion mining: the case of the energy industry with long-term coal R\&D investment projects. J. Intell. Stud. Bus. 8(2), 6-22 (2018)

30. Huang, Q., Cervone, G., Zhang, G.: A cloud-enabled automatic disaster analysis system of multi-sourced data streams: an example synthesizing social media, remote sensing and Wikipedia data. Comput. Environ. Urban Syst. 66, 23-37 (2017)

31. Basavaraju, P., Varde, A.: Supervised learning techniques in mobile device apps for Androids. ACM SIGKDD Explor. 18(2), 18-29 (2016)

32. Persaud, P., Varde, A., Robila, S.: Enhancing autonomous vehicles with commonsense: smart mobility in smart cities. In: IEEE ICTAI, pp. 1008-1012, November 2017

33. Pandey, A., Puri, M., Varde, A.: Object detection with neural models, deep learning and common sense to aid smart mobility. In: IEEE ICTAI, pp. 859-863, November 2018

34. Packt Publishing: Artificial Intelligence for Smart Cities. Becoming Human: AI Magazine. https://becominghuman.ai/artificial-intelligence-for-smart-cities-64e6774808f8

35. Garg, A., Tandon, N., Varde, A.: I am guessing you can't recognize this: generating adversarial images for object detection using spatial commonsense. In: AAA Conference, February 2020 